\title{
Screening for Early Detection of Cervical Cancer in Women Living with HIV in Mumbai, India - Retrospective Cohort Study from a Tertiary Cancer Center
}

\author{
Sharmila A. Pimple ${ }^{10}$ Vandita Pahwa ${ }^{10}$ Gauravi A. Mishra1이 Kavita V. Anand ${ }^{10}$ \\ Saleem Pathuthara2 ${ }^{20}$ Sanjay K. Biswas ${ }^{30}$
}

${ }^{1}$ Department of Preventive Oncology, Centre for Cancer Epidemiology (CCE), Tata Memorial Centre, Homi Bhabha National Institute, Mumbai, Maharashtra, India

2 Department of Microbiology, Tata Memorial Centre, Homi Bhabha National Institute, Mumbai, Maharashtra, India

${ }^{3}$ Department of Microbiology, Tata Memorial Centre, Homi Bhabha National Institute, Mumbai, Maharashtra, India

Address for correspondence Dr. Sharmila A. Pimple, MD (PSM), Department of Preventive Oncology, Tata Memorial Centre, Homi Bhabha National Institute, 3rd Floor, Service Block, E. Borges Marg, Parel, Mumbai, 400 012, Maharashtra, India (e-mail: drsharmilapatil@yahoo.com; pimplesa@tmc.gov.in).

\begin{abstract}
Keywords

- Human Papilloma Virus

- Human immunodeficiency virus

- cervical cancer screening

Introduction Women living with human immunodeficiency virus (HIV) have an increased risk of persistent human papillomavirus infection (HPV) of developing cervical cancer precursors and are, therefore, considered at higher risk for cervical cancer. Despite the higher risk, screening for cervical cancer is extremely low among HIV-positive women in India.

Objectives Given the limited usefulness of cytology-based screening programs, the current study retrospectively evaluated the comparative performance of visual inspection with $5 \%$ acetic acid (VIA), conventional cytology, and human papillomavirus (HPV) testing among HIV-positive women attending the cancer screening clinic at the tertiary cancer center.

Materials and Methods Retrospective analysis of 291 HIV-positive women attending cervical cancer screening services in a tertiary cancer center in Mumbai was undertaken. All underwent simultaneous screening with VIA, Pap cytology, and HPV DNA testing, followed by diagnostic colposcopy and histopathology. Sensitivity, specificity, positive predictive value (PPV), and negative predictive value (NPV) to detect cervical intraepithelial neoplasia (CIN) 2/3 on histology were estimated.
\end{abstract}

\section{Introduction}

Cervical cancer despite being one of the most preventable cancers is the fourth most common cause of cancer inci- dence and mortality in women worldwide. ${ }^{1}$ Persistent infection with high-risk human papillomavirus infection (hrHPV) is a necessary precondition for the development of all cervical cancer and precancerous intraepithelial
DOI https://doi.org/ $10.1055 / \mathrm{s}-0042-1742662$. ISSN 0971-5851. (c) 2022. Indian Society of Medical and Paediatric Oncology. All rights reserved.

This is an open access article published by Thieme under the terms of the Creative Commons Attribution-NonDerivative-NonCommercial-License, permitting copying and reproduction so long as the original work is given appropriate credit. Contents may not be used for commercial purposes, or adapted, remixed, transformed or built upon. (https://creativecommons.org/ licenses/by-nc-nd/4.0/)

Thieme Medical and Scientific Publishers Pvt. Ltd., A-12, 2nd Floor, Sector 2, Noida-201301 UP, India 
Results The screen positivity rate for cervical cancer screening by VIA, high-risk HPV DNA, and Pap cytology was 35.7, 34.4, and 6.2\% respectively. At the CIN2+ disease threshold, the sensitivity, specificity, PPV, and NPV estimates were $80.00 \%$ (59.3093.17), 68.42\% (62.46-73.96), 19.23\% (15.46-23.67), 97.33\% (94.30-98.77) for VIA; 80.00\% (68.78-97.45), 70.68\% (64.81-76.08), 22.00\% (18.22-26.32), 98.43\% (95.5899.45) for HPV DNA; and 64.00\% (42.52-82.03), 98.12\% (95.67-99.39), 76.19\% (56.13-88.89), 96.67\% (94.50-98.00) for cytology (HSIL cutoff).

Conclusion The diagnostic performance of VIA and HPV DNA was comparable and better than cytology indicating that VIA as a cost-effective cervical cancer screening test can be incorporated within the services under sexually transmitted diseases /HIV testing and counseling centers within the country.

lesions and is one of the most common sexually transmitted infections worldwide. ${ }^{2}$

In India, the burden of cervical cancer is high, with over a quarter of global cervical cancer deaths occurring in India. The age-standardized rate prevalence of cervical cancer is $>15.2$ per 100,000 women in India with mortality reported being $9.0-16.4$ per 100,000 women. ${ }^{3}$ Women having sexually transmitted disease (STD) are at an increased risk for cervical cancer. Immunosuppressed status, certain lifestyle, and reproductive and high-risk behaviors such as multiple sexual partners increase a woman's risk for contracting STD, which can lead to cervical cancer. Human immunodeficiency virus (HIV)-infected women are one of the high-risk groups, to acquire an HPV infection, which is likely to be more persistent and progress rapidly to cervical intraepithelial neoplasia (CIN) and invasive cervical cancer (ICC). ${ }^{4}$

Women living with HIV infection too are at higher risk for cervical cancer-an AIDS (Acquired immunodeficiency syndrome)-defining diagnosis. Cervical cancer is the most frequently detected cancer in women living with HIV and is classified as an AIDS-defining illness. It has been well known that HIV infection increases the risk of developing certain cancers, and Kaposi sarcoma, non-Hodgkin lymphoma, and cervical cancer have been classified as AIDS-defining diseases. $^{5}$

Women infected with HIV have an increased risk of being infected with HPV, persistent infection with high-risk types of HPV, and developing cervical cancer precursors and are, therefore, considered at higher risk for cervical cancer. ${ }^{6}$ As per the literature, HIV acquisition increases by twofold among women with HPV infection. The behavioral risk factors for the two infections are the same. ${ }^{7,8}$ As per the recent estimates of the global burden of cervical cancer associated with HIV, the likelihood that a woman living with HIV will develop ICC is up to six times higher when compared to women without HIV. Worldwide, an estimated $5 \%$ of all cervical cancer cases are attributable to HIV. ${ }^{9}$ However, there were wide disparities across countries and regions of the world. HIV estimations for 2019 in India have reported that HIV-infected women (for $15+$ years) constituted around $44 \%$ of the total 23.49 lakh people living with HIV (PLHIV). ${ }^{10}$ The data for cervical cancer deaths among the HIV cohort is inconsistent. ${ }^{11-14}$ Improved access to antiretroviral therapy has increased survival and improved longevity in many parts of the world including India. However, most women lack access to cervical cancer screening services.

The three primarily used screening methods of conventional cytology (Papanicolaou/Pap smear), hrHPV testing, and visual inspection with acetic acid (VIA) have been widely researched and evaluated for accuracy and efficacy in different settings. ${ }^{15-18}$ However, the evidence assessing the test accuracy of these screening tools among HIV-infected women is sparse and remains largely undocumented. Comparative estimates of available screening technologies are needed for providing a better understanding of the most appropriate strategy to screen HIV-infected women. The feasible process of integrating cervical cancer screening tools in the STD/HIV/ AIDS testing centers technically known as integrated counseling and testing centers (ICTC) located in government hospitals in India needs to be evaluated. These centers provide a critical entry point to individuals practicing high-risk behavior or likely to have a higher prevalence of STD/HIV/AIDS. However, in most public STD clinics, cervical cancer screening is not part of the routine testing offered to women attending the ICTC. The current study retrospectively sought to evaluate an accurate, affordable, and feasible method to screen HIV-infected women for prevention and early detection of cervical cancer among HIV-positive women attending the cancer screening clinic at the tertiary cancer center.

\section{Methodology}

This is a retrospective cohort study of HIV-positive women who received cervical cancer screening at the preventive oncology cancer screening clinic at a tertiary cancer care institute between May 2010 and June 2015. The department collaborated with non-governmental organizations (NGOs) working for HIV-positive patients and carried out activity for screening HIV-positive women for common cancers in India. The mobilization of HIV-positive women to the hospital was undertaken by respective NGOs. The department also undertook cervical cancer screening, diagnostic work up with treatment for walk-in patients, and referred HIV-positive women. The cancer screening clinic routinely collected relevant information from patients including demographic 
The department collaborated with NGOs working for HIV-positive patients and had cervical cancer awareness sensitization sessions for them

\section{$\downarrow$}

HIV-positive women were mobilized by the respective NGOs to the preventive oncology screening clinic

The cancer screening clinic routinely explained the procedure, took consent, collected relevant information from patients' including demographic characteristics, reproductive, and medical history and carried out routine cervical cancer screening tests.

\section{$\downarrow$}

All HIV-positive women were simultaneously administered conventional cervical cytology, HPV testing was done by the Digene Hybrid Capture-2TM assay (Qiagen, Gaithersburg, MD), and visual inspection was done after the application of dilute (3-5\%) acetic acid on the cervix (VIA) sequentially.

Cervical pre-cancer or cancer received treatment as per the institutional evidence-based management protocol.

Fig. 1 Routine flowcharts of HIV-positive patients in preventive oncology screening clinic.

characteristics, reproductive, and medical history and carried out routine cervical cancer screening tests. HIV-infected women underwent VIA, cytology, HPV testing, and diagnostic colposcopy as part of the standard cervical cancer screening tests offered at the preventive oncology screening clinic (-Fig. 1).

\section{Retrieval of Data}

Case records of HIV-positive women registered from January 1, 2010 to December 31, 2015 were identified and retrieved from the electronic medical records (EMRs) of the hospital information system. A total of 312 records of laboratory-diagnosed HIV-positive women registered for the preventive oncology screening clinic were identified and retrieved through the central EMR system of the hospital. The confidentiality and identity of patients were protected by generating a unique identification code (UIC) to all the records. All patient identifiers such as name, address, telephone numbers, and contact details if any were removed. The de-identified records were then used to capture data as per the study objectives on a standardized structured data capture format. Following inclusion criteria were applied to the selected participant records to be included for the retrospective analysis: (a) female patients aged 21 years and above, (2) laboratory diagnosed HIV-positive status record, (3) HIV-positive women who underwent cervical cancer screening by VIA, conventional cytology, and HPV DNA HC II test, and (4) HIV-positive women who underwent diagnostic evaluation by colposcopy.

All the 312 digital records were further scanned for completeness for future screening and diagnostic tests. Out of the total 312 records, digital records of 291 women who met the inclusion criteria were included for analysis. Patient records of HIV-positive women who had not undergone all the three cervical cancer screening tests with incomplete screening and diagnostic investigation of colposcopy were excluded from the analysis. The hospital EMR system contains the reports of all screening and diagnostic tests performed at the screening clinic. Information on HIV and antiretroviral therapy (ART), most recent CD4+ cell counts, was obtained from the participant's medical record. The EMR of these patients were examined, and demographic details and information of the screening tests, histology was retrieved and input in a standardized data collection form. 


\section{Cervical Cancer Screening Tests, Diagnostic Colposcopy, and Histopathology}

As part of the standard cervical cancer screening protocol for HIV-positive women registered for cervical cancer screening clinic, all 291 women were simultaneously administered conventional cervical cytology, HPV testing by the Digene Hybrid Capture-2TM (HC2) assay (Qiagen, Gaithersburg, MD, United States), and visual inspection after application of dilute (3-5\%) acetic acid on the cervix (VIA) sequentially. Diagnostic colposcopy was undertaken for all women irrespective of their screening test result. Colposcopy-guided punch biopsies for histopathology evaluation were obtained only from women with abnormal findings on colposcopy. VIA was scored positive when a well-defined, dense acetowhite area abutting or touching the squamocolumnar junction in the transformation zone was observed as per International Agency for Research on Cancer/World Health Organization (WHO) guidelines. ${ }^{19}$ All the tests were administrated by gynecologists or the post-MD preventive and social medicine registrars who had completed 1 year of training and service in the department. Pap cytology test results were reported according to the Bethesda system. ${ }^{20}$ Histopathology was reported using the CIN system. True disease status was defined as CIN-2 grade and worse lesions. ${ }^{21}$ The gold standard for the diagnosis of true disease was based on histology and negative colposcopy, thereby minimizing the verification bias of the screening test performance parameters. Women diagnosed with invasive cancer were referred for further management to the gynecology department of the institute. Cervical pre-cancer or cancer received treatment as per the institutional evidence-based management protocol. ${ }^{22}$

Primary outcome measures of prevalence of high-risk HPV infection and CIN among HIV-positive women were estimated. Among the secondary outcomes measures, screening test positivity rates of the three primary cervical cancer screening tests were estimated and test accuracy performance of the screening tests was determined by deriving comparative estimates of sensitivity, specificity, positive predictive values (PPVs), and negative predictive values (NPVs).

All the standard precautions were followed while handling infectious materials. Handwashing before wearing gloves and after the examination was done. All aseptic precautions were taken. Double gloves were used by the doctors attending the patients. Protective plastic gowns and protective eye goggles were used during the examination. Immediately after use, all instruments were placed in $0.5 \%$ chlorine solution for 10 minutes. Instruments were then cleaned with a brush in detergent water to remove blood, other body fluids, organic material, tissue, and dirt and sent for autoclaving. For decontaminating the surfaces of the examination/procedure table, parts of any equipment/instrument that may have come in contact with body fluids, $0.5 \%$ chlorine solution or $90 \%$ ethyl alcohol was used for wiping the surfaces.

\section{Statistical Analysis}

Data were analyzed using IBM SPSS Statistics v 24.0 (SPSS/IBM, Chicago, IL, United States). Screening test positiv- ity rates were presented as numbers and proportions. Detection rates of CIN were presented as the proportion of screenpositive CIN detected among the screened women. Screening tests accuracy parameters of sensitivity, specificity, PPVs, and NPVs and their exact 95\% confidence intervals (CIs) were calculated using standard formulae. The ethics committee approval was sought for undertaking the retrospective analysis of the cohort of HIV-positive women enrolled in the preventive oncology screening clinic of the tertiary cancer care hospital.

\section{Ethics}

The retrospective study audit was conducted in accordance with the Declaration of Helsinki and Good Clinical Practices as defined by the International Conference on Harmonization. The study was conducted in compliance to the protocol. The retrospective study protocol had received the approval (Project ID: 3875, dated 29 December 2021) of the Tata Memorial Centre Institutional Ethics Committee. Subject confidentiality was maintained at all times by de-identification of patient records. The identity of the patients was protected by generating UIC for each of those patients whose data were retrieved for analysis as per the study protocol. Waiver for informed consent was obtained from the ethics committee.

\section{Results}

Total 291 HIV-infected women were evaluated with conventional cytology, HPV HC2, and VIA for cervical cancer screening. The sociodemographic profile of the women is as shown in - Table 1. The age group from 30 to 49 years included $77.7 \%$ of the women with a mean age of 35.91 years. Middle and high school education was received by $61.8 \%$ women. Marital status reflected $50.8 \%$ as widowed/separated with the mean age of marriage being 20.6 years. With respect to HIV characteristics, the majority of the women (57.8\%) were HIV positive beyond 5 years duration, spouse's HIV status was positive for $81.4 \%$ of the women, and $74.2 \%$ were currently on ART treatment.

Among the cervical cancer screening tests modalities administered to the HIV-positive women, VIA and HPV HC2 test positivity rates were demonstrated to be $35.7 \%$ and $34.4 \%$, respectively. A total of $20.6 \%(n=60 / 291)$ women in the study population demonstrated epithelial cell abnormalities on cytology. Atypical squamous cells of undetermined significance (ASCUS) was demonstrated in 3.8\% $(n=291)$, low-grade squamous intraepithelial lesion (LSIL) in $9.6 \%(n=291)$, high-grade squamous intraepithelial lesion (HSIL) in $6.2 \%(n=291)$, and invasive carcinoma in $1 \%$ $(n=291)$. Histopathology results were available for 114 / 291 (39.2\%), for the rest diagnostic colposcopic impressions served as a final diagnosis. In the current study, $1.7 \%$ women were diagnosed with invasive cancer on colposcopy and all these women were within stage $1 \mathrm{~A} 2$. The composite gold standard of colposcopy-histopathology diagnosis confirmed 18 (6.2\%) CIN I, 13 (4.5\%) CIN II, and 12 (4.1\%) CIN III and above lesions (-Table 2 ). 
Table 1a Sociodemographic variation among HIV-positive women in Mumbai, India $(n=291)$

\begin{tabular}{|c|c|c|c|}
\hline \multicolumn{2}{|c|}{ Sociodemographic variables } & \multirow{2}{*}{$\begin{array}{l}\text { Frequency }(n) \\
291 \\
\end{array}$} & \multirow{2}{*}{$\begin{array}{l}\text { Percent (\%) } \\
100.0 \\
\end{array}$} \\
\hline \multirow{5}{*}{ Ages } & & & \\
\hline & $<30$ & 50 & 17.2 \\
\hline & $30-39$ & 156 & 53.6 \\
\hline & $40-49$ & 70 & 24.1 \\
\hline & $50-64$ & 15 & 5.2 \\
\hline \multirow[t]{4}{*}{ Education } & Nil & 81 & 27.8 \\
\hline & Primary & 30 & 10.3 \\
\hline & Middle & 58 & 19.9 \\
\hline & High school and above & 122 & 41.9 \\
\hline \multirow[t]{3}{*}{ Occupation (Self) } & Professional/semi-professional & 33 & 11.3 \\
\hline & Skilled/semi-skilled/ unskilled worker & 64 & 22.0 \\
\hline & Housewife & 194 & 66.7 \\
\hline \multirow[t]{3}{*}{ Spouse's occupation } & Semi-professionals & 43 & 14.8 \\
\hline & Unskilled worker & 155 & 53.3 \\
\hline & Unemployed & 93 & 32.0 \\
\hline \multirow[t]{3}{*}{ Age of menarche } & $10-12 y$ & 65 & 22.3 \\
\hline & $13-15 y$ & 190 & 65.3 \\
\hline & $>15 y$ & 36 & 12.4 \\
\hline \multirow[t]{3}{*}{ Marital status } & Married & 143 & 49.1 \\
\hline & Widowed & 134 & 46.0 \\
\hline & Separated & 14 & 4.8 \\
\hline \multirow[t]{5}{*}{ Age married } & $<15$ & 46 & 15.8 \\
\hline & $16-20$ & 164 & 56.4 \\
\hline & $21-25$ & 51 & 17.5 \\
\hline & $26-30$ & 22 & 7.6 \\
\hline & $>30$ & 8 & 2.7 \\
\hline \multirow[t]{3}{*}{ Pregnancies } & $\leq 2$ & 154 & 52.9 \\
\hline & $3-5$ & 128 & 44.0 \\
\hline & $>5$ & 9 & 3.1 \\
\hline \multirow[t]{3}{*}{ Abortions } & $\leq 2$ & 276 & 94.8 \\
\hline & $3-5$ & 13 & 4.5 \\
\hline & $>5$ & 2 & 0.7 \\
\hline \multirow[t]{2}{*}{ Tobacco use } & Yes & 62 & 21.3 \\
\hline & No & 229 & 78.7 \\
\hline
\end{tabular}

Abbreviation: HIV, human immunodeficiency virus.

\section{Screening Test Characteristics of the Three Cervical Cancer Screening Tests:}

The sensitivity, specificity, and PPVs for VIA to detect CIN2 and 3+ lesions were 80.00\% [59.30-93.17], 68.42\% [62.46-73.96], and 19.23\% [15.46-23.67]; the corresponding values for HPV HC II were $80.00 \%$ [68.78-97.45 ], 70.68\% [64.81-76.08 ], and $22.00 \%$ [18.22-26.32 ]; for cytology at LSIL threshold were 88.00\% [68.78-97.45], 89.85\% [85.58-93.20], and 44.90\% [35.65-54.51] and for cytology HSIL + threshold was 64.00\%
[42.52-82.03], 98.12\% [95.67-99.39], and 76.19\%[56.13-88.89], respectively ( $\mathbf{- T a b l e ~} 3$ ). Colposcopy evaluation by Swedes scoring as per nomenclature adopted by the International Federation for Cervical Pathology and Colposcopy ${ }^{23}$ showed the corresponding values for sensitivity, specificity, and PPVs at 88.00\% [68.78-97.45], 75.56\% [69.94-80.61], 25.29\% [20.7630.42] for the threshold of CIN 1 and above lesions and $60.00 \%$ [38.67\%- 78.87], 98.50\% [96.29-99.59], 78.95\% [57.39\%-91.26] for the threshold of CIN 2 and above lesions. 
78 Screening for CIN among HIV-Infected Women Pimple et al.

Table 1b HIV demographic variation among HIV-positive women in Mumbai, India $(n=291)$

\begin{tabular}{|c|c|c|c|}
\hline \multicolumn{2}{|l|}{ HIV demographic variables } & \multirow{2}{*}{$\begin{array}{l}\text { Frequency }(n) \\
291\end{array}$} & \multirow{2}{*}{$\begin{array}{l}\text { Percent (\%) } \\
100.0\end{array}$} \\
\hline Total & & & \\
\hline \multirow[t]{2}{*}{ Spouse's HIV status } & Positive & 237 & 81.4 \\
\hline & Negative & 54 & 18.6 \\
\hline \multirow[t]{6}{*}{ Duration spouse has been HIV positive } & Not HIV positive & 54 & 18.6 \\
\hline & $\leq 1 \mathrm{y}$ & 68 & 23.4 \\
\hline & $1-5 y$ & 49 & 16.8 \\
\hline & $5-10 y$ & 71 & 24.4 \\
\hline & $10+y$ & 22 & 7.6 \\
\hline & Unknown & 27 & 9.3 \\
\hline \multirow[t]{4}{*}{ Modality of HIV transmission } & Sexual transmission & 201 & 69.1 \\
\hline & Blood transfusion & 18 & 6.2 \\
\hline & Infected needles & 8 & 2.7 \\
\hline & Unknown & 64 & 22.0 \\
\hline \multirow[t]{4}{*}{ Duration of HIV-positive status } & $<1 \mathrm{y}$ & 25 & 8.6 \\
\hline & $1-5 y$ & 98 & 33.7 \\
\hline & $5-10 y$ & 125 & 43.0 \\
\hline & $>10 y$ & 43 & 14.8 \\
\hline \multirow[t]{2}{*}{ ART treatment } & Yes & 216 & 74.2 \\
\hline & No & 75 & 25.8 \\
\hline \multirow[t]{4}{*}{ Duration on ART Rx } & Not applicable & 75 & 25.8 \\
\hline & $\leq 5$ & 148 & 50.9 \\
\hline & $5-10$ & 49 & 16.8 \\
\hline & $>10$ & 19 & 6.5 \\
\hline \multirow[t]{4}{*}{ Other treatment modality } & Homeopathy & 16 & 5.5 \\
\hline & Ayurvedic & 11 & 3.8 \\
\hline & Other & 3 & 1.0 \\
\hline & None & 261 & 89.7 \\
\hline \multirow[t]{4}{*}{ Allied medical ailments } & T.B. & 89 & 30.6 \\
\hline & General (weakness/pain) & 23 & 7.9 \\
\hline & Other & 5 & 1.7 \\
\hline & None & 174 & 59.8 \\
\hline \multirow[t]{4}{*}{ CD4 count } & $<200$ & 26 & 8.9 \\
\hline & $200-499$ & 74 & 25.4 \\
\hline & $\geq 500$ & 44 & 15.1 \\
\hline & Unknown & 147 & 50.5 \\
\hline
\end{tabular}

Abbreviations: ART, antiretroviral therapy; HIV, human immunodeficiency virus; T.B., tuberculosis.

\section{Discussion}

Women living with HIV are at increased risk for HPV infection including CIN2/3 and ICC. ${ }^{24,25}$ Substantial progress has been achieved with the availability of highly active antiretroviral therapy, with decrease in morbidity and mortality associated with HIV infection in both higher- and lowermiddle-income countries. Despite improved survival and longevity, women living with HIV/AIDS (WLWHA) continue to be affected by gynecologic and non-gynecologic malignancies at disproportionate rates in lower-middle-income country settings, especially with high HIV burden. This is due to the lack of effective cervical cancer prevention programs. ${ }^{26}$ In our retrospective study, we examined the prevalence of HPV infection, screening positivity rates of screening methods of VIA, HPV HC II, and cytology among HIV-infected 
Table 2 Distribution of the histopathological results stratified by the findings of various screening tests performed in HIV-positive women $(n=291)$

\begin{tabular}{|c|c|c|c|c|c|c|c|c|c|c|c|}
\hline \multirow{4}{*}{ Screening test } & & & & \multicolumn{8}{|c|}{ HPR } \\
\hline & & & & \multicolumn{2}{|c|}{ Benign } & \multicolumn{2}{|c|}{ CIN I } & \multicolumn{2}{|c|}{ CIN II } & \multicolumn{2}{|c|}{ CIN III + } \\
\hline & & $n$ & (\%) & $n$ & $(\%)$ & $n$ & (\%) & $n$ & $(\%)$ & $n$ & (\%) \\
\hline & & 291 & & 248 & 85.20 & 18 & 6.20 & 13 & 4.50 & 12 & 4.10 \\
\hline \multirow[t]{2}{*}{ VIA } & Positive & 104 & 35.7 & 69 & 66.30 & 15 & 14.40 & 11 & 10.60 & 9 & 8.70 \\
\hline & Negative & 187 & 64.3 & 179 & 95.70 & 3 & 1.60 & 2 & 1.10 & 3 & 1.60 \\
\hline \multirow[t]{2}{*}{ HPV HC II } & Positive & 100 & 34.4 & 66 & 66.00 & 12 & 12.00 & 12 & 12.00 & 10 & 10.00 \\
\hline & Negative & 191 & 65.6 & 182 & 95.30 & 6 & 3.10 & 1 & 0.50 & 2 & 1.00 \\
\hline \multirow{6}{*}{$\begin{array}{l}\text { Conventional } \\
\text { cytology }\end{array}$} & Normal & 129 & 44.3 & 127 & 98.40 & 2 & 1.60 & 0 & 0.00 & 0 & 0.00 \\
\hline & Inflammation & 102 & 35.1 & 95 & 93.20 & 4 & 3.90 & 1 & 1.00 & 2 & 2.00 \\
\hline & ASCUS & 11 & 3.80 & 8 & 72.70 & 3 & 27.30 & 0 & 0.00 & 0 & 0.00 \\
\hline & LGSIL & 28 & 9.60 & 14 & 50.00 & 8 & 28.60 & 4 & 14.30 & 2 & 7.10 \\
\hline & HGSIL & 18 & 6.20 & 4 & 22.30 & 1 & 5.60 & 8 & 44.40 & 5 & 27.80 \\
\hline & Invasive cancer & 3 & 1.00 & 0 & 0.00 & 0 & 0.00 & 0 & 0.00 & 3 & 100 \\
\hline \multirow{5}{*}{$\begin{array}{l}\text { Colposcopy } \\
\text { Findings }\end{array}$} & Normal & 140 & 48.11 & 138 & 98.57 & 1 & 0.71 & 1 & 0.71 & 0 & 0.00 \\
\hline & Probable low-grade lesion (CIN I) & 69 & 23.71 & 48 & 69.60 & 14 & 20.30 & 5 & 7.20 & 2 & 2.90 \\
\hline & Probable high-grade lesion (CIN II + ) & 14 & 4.80 & 2 & 14.30 & 2 & 14.30 & 6 & 42.90 & 4 & 28.60 \\
\hline & Invasive carcinoma & 5 & 1.70 & 0 & 0.00 & 0 & 0.00 & 1 & 20.00 & 4 & 80.00 \\
\hline & Other Inconclusive /Unsatisfactory & 63 & 21.60 & 60 & 95.20 & 1 & 1.60 & 0 & 0.00 & 2 & 3.20 \\
\hline
\end{tabular}

Abbreviations: ASCUS, atypical squamous cells of undetermined significance; CIN, cervical intraepithelial neoplasia; HGSIL, high-grade squamous intraepithelial lesions; HIV, human immunodeficiency virus; HPR, histopathology report; HPV HC II, human papillomavirus hybrid capture 2; LSIL, low-grade squamous intraepithelial lesions; VIA, visual inspection with acetic acid.

women. Our study findings have provided useful comparable measures of evaluation of the three cervical cancer screening tools for the most feasible and effective screening strategies among HIV-positive women that can be chosen for implementation in public health program settings.

The three screening tests administered to HIV-infected women showed very high screening test positivity rates of $35.7 \%, 34.4 \%$ for VIA and HPV HC2, and 9.6\% for cytology (LSIL). Comparative screening test positivity rates among HIV-positive women from other studies in India have reported $15.0 \%$ for VIA, $8.1 \%$ for any abnormal cytology, and $26.4 \%$ for HPV. ${ }^{27}$ In a study from eastern India, among the 216 HIV-positive women screened, $26.85 \%$ were HPVpositive, $17.11 \%$ had LSIL, $5.35 \%$ had HSIL, and three (1.60\%) had carcinoma cervix. ${ }^{28}$ In a study from Ethiopia in Africa, $22.1 \%$ were found to be positive on VIA. ${ }^{29}$ A study from South Africa reported that HIV-positive women were more likely to have an abnormal cytology result as compared to HIV-negative women (35.1\%, $[n=20 / 57]$ vs. $13.9 \%$, $[n=31 / 223] ; p=0.0002)$ and specifically a higher proportion of HSIL (10.5\%, [ $n=6 / 57]$ vs. 3.1\%, $[n=7 / 223]$; $p=0.028) .{ }^{30}$ Wide variations in the screening test positivity rates among HIV-positive women are largely explained by the provider. They explained the dependent, subjective nature of the VIA test, whereas cytology could be impacted right from adequate collection of the sample to the level of training and nature of quality assurance measures adopted in the respective laboratories for conventional cytology. Out of the three tests, molecular high-risk HPV detection is much less dependent on the quality of the sample and on human judgment than are cytology and visual inspection. The molecular laboratory requirements and overall cost of the test are still very prohibitive for scale-up in lowresource countries.

The prevalence of all categories $\operatorname{CIN}$ (CIN 1,2,3) detected in our study was $14.7 \%$. The prevalence of CIN2 and above lesions was $8.6 \%$ which was slightly higher than some of the cross-sectional studies among HIV-infected women. Cross-sectional studies from Kenya, ${ }^{31}$ Brazil, $^{32}$ and southwestern China ${ }^{33}$ reported the prevalence of CIN 2/3 as 5.4, 6.0 , and $8.4 \%$, respectively. Two studies from the same region in Maharashtra, India, however, have shown wide variation in the prevalence of $\mathrm{CIN} 2 / 3$ lesions at $4.9 \%$ with more than 1,000 HIV-positive women ${ }^{27}$ and $16 \%$ with much smaller sample size of 303 women. ${ }^{34}$ Some other studies ${ }^{35-37}$ including those from India 28,38 have demonstrated a higher prevalence of CIN and cervical cancer among HIV-infected women. HIV-infected women invariably are one of the highrisk groups for the acquisition, persistence, progression, and recurrence of HPV infection and thereby HPV-induced cervical precursor lesions and cervical cancer. Thus, there is a need for updated revised guidelines for more frequent screening and greater follow-up among HIV-infected women than HIV-uninfected women. 


\begin{tabular}{|c|c|c|c|c|c|c|}
\hline 足 & 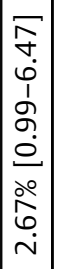 & 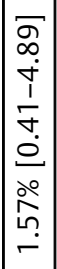 & 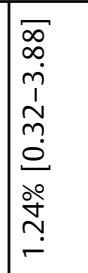 & 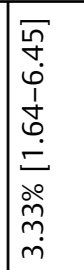 & 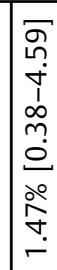 & 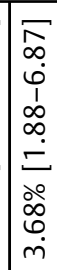 \\
\hline 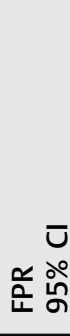 & 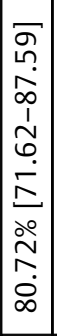 & 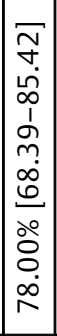 & 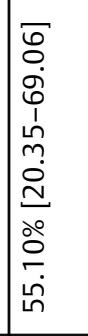 & 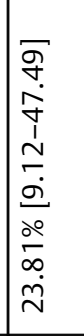 & 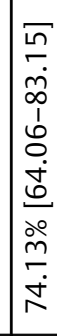 & 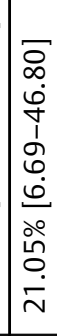 \\
\hline 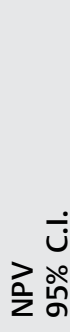 & 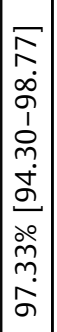 & 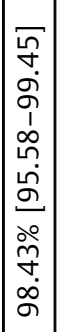 & 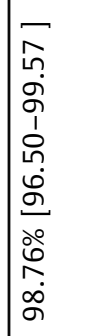 & 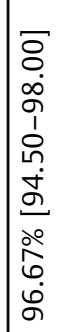 & 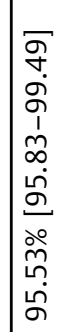 & 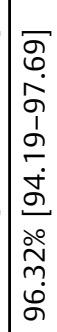 \\
\hline 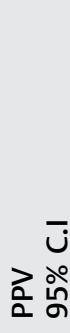 & 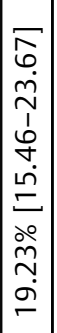 & 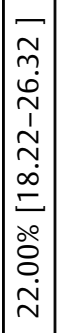 & 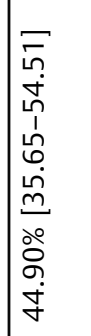 & 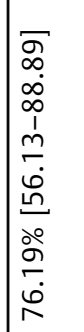 & 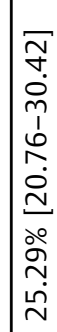 & 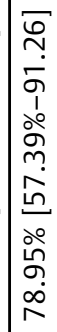 \\
\hline 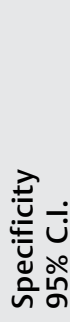 & 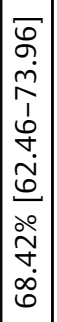 & 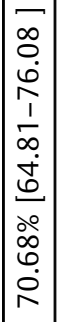 & 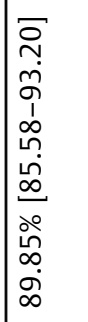 & 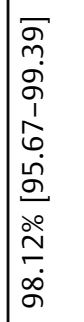 & 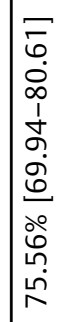 & 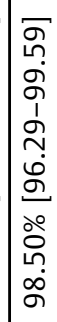 \\
\hline 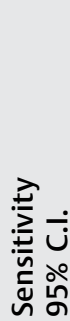 & 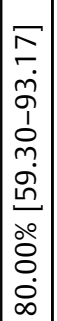 & 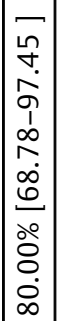 & 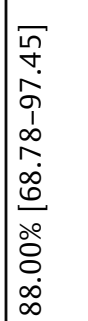 & 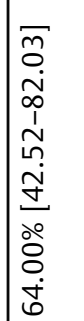 & 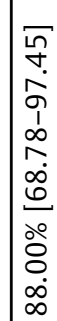 & 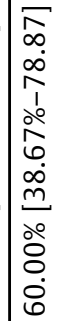 \\
\hline & 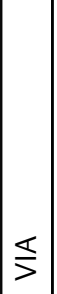 & 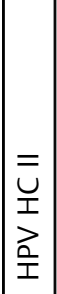 & 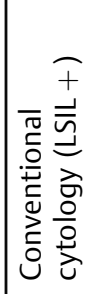 & 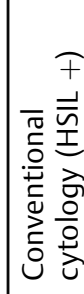 & 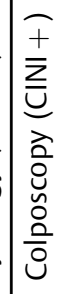 & 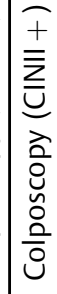 \\
\hline
\end{tabular}

With respect to the comparative screening test performance characteristics for screening of cervical cancers, multiple studies in resource-limited settings have extensively evaluated the performance of VIA, HPV, and cytology in the general population. ${ }^{39,40}$ Our study is one of the very few which has concurrently evaluated the screening test performance of three screening tests including diagnostic colposcopy among HIV-infected women in detecting CIN 2 and 3 lesions with minimal verification bias. The study has demonstrated the use of diagnostic colposcopy and, if indicated, histopathology to investigate the true prevalence of CIN in this high-risk cohort of women. With the gold standard of diagnostic colposcopy, with or without biopsy, our results indicate that VIA, HPV testing, and Pap cytology (LSIL + ) have a higher sensitivity in detecting high-grade CIN 2 and above lesions. Pap cytology (HSIL + ) and diagnostic colposcopy $(\mathrm{CINII}+)$ showed low sensitivity but high specificities with very high PPVs.

Screening test performances among general women in developing country settings, the sensitivity of VIA to detect high-grade precursor lesions and ICC has varied from 49 to $96 \%$ and the specificity from 49 to $98 \%$. This is largely due to the subjective nature of the tests and requirements for maintaining adequate training levels of the providers with good-quality assured test procedures. ${ }^{39,40} \mathrm{HPV}$-DNA sensitivity was high (66-100\%) with moderate specificity (61$96 \%$ ). Although it has the special advantage of being high throughput, objective, reproducible, and robust, it, however, requires molecular laboratory platforms and is currently expensive. Conventional cytology demonstrated moderate sensitivity (44-78\%) but high specificity (91-96\%) and requires adequate health care infrastructure, laboratorybased, stringent training, and quality control measures. Colposcopy sensitivity was low (44-77\%), with moderate specificities (85-90\%), and is generally expensive to source and maintain with good clinical training skill requirements to perform it. ${ }^{39,40}$

Performances of the three screening tests among the HIVpositive women population showed comparable measures of accuracy of performing these tests. In a study of cervical cancer screening among HIV-positive women in India, sensitivity for VIA, cytology at ASCUS threshold, and HPV testing was 83.6, 63.3, and 94.6\%, and specificity in detecting CIN2/3 lesions was $88.8,94.5$, and $77.4 \%$, respectively. ${ }^{27}$ Another study from western India which evaluated VIA and cervical cytology among HIV-positive women with composite disease ascertainment by colposcopy and/or histopathology, the sensitivity, specificity, and PPV, and NPV estimates at the CIN2 and above disease threshold for VIA were 80, 82.6, 47.6, and 95.4 respectively, compared to $60.5,64.6,24.8$, and $89.4 \%$ for LSIL and above cutoff on cytology, and 20.9, 96.0, 50.0, $86.3 \%$ for HSIL and above cutoff on cytology, respectively. ${ }^{34} \mathrm{~A}$ study from Nigeria reported sensitivity, specificity, and PPV of VIA as 76.0, 83.0, and $34.0 \%$ and those of cervical cytology as $57.0,95.0$, and $55.0 \%$ (95\% CI 33.0-75.0), respectively. ${ }^{41}$ Our study findings add to the existing available literature by providing evidence on comparable test characteristics of three screening modalities and independent diagnostic 
assessment by colposcopy on limiting verification bias in resource-constrained settings. Here, histopathology services are not always available.

The incidence of cervical cancer is high among HIVinfected women, particularly in countries where there are no organized cervical cancer prevention programs. HPVrelated cancers are likely to remain an important problem in HIV-infected women for the foreseeable future, even among those on effective ART. ${ }^{42}$ In the call to action to eliminate cervical cancer as a public health problem, WHO has initiated Guideline Development Group for "Initiation and frequency of screening of cervical pre-cancer lesions in women with HIV" in March 2020. Reaching vulnerable women with HIV infection at the high risk of developing cervical cancer will need prioritization of preventive screening and treatment services to be integrated with STD/HIV testing centers. In countries which are low on resources, the screening intervals will depend on financial, infrastructural, and other resources. The guideline indicates that the screening interval for repeat screening should be within 3 years for women who are of HIV-positive status or unknown HIV status in areas with high endemic HIV infection. Our study results provide good evidence of the feasibility of VIA as a primary screening modality for ease and cost of administration.

In a first "real-world" demonstration in a public sector, implementation program in Zambia, in sub-Saharan Africa, successfully showed the integration of delivering cervical cancer screening services, in vertical health initiatives like HIV/AIDS care programs as a routine health care service. ${ }^{43}$ Similar efforts of the exploration of adoption and implementation of cervical cancer screening in STD clinics were successfully demonstrated by strategical adaptation of addressing staff concerns, training, specific protocol adaptation, and resource allocation to facilitate greater staff adoption of cervical cancer screening. ${ }^{44}$ Centers for Disease Control and Prevention, United States, has provided algorithms for screening and management of women for STD clinics offering cervical screening services to have protocols in place for follow-up of test results and referral. ${ }^{45}$

\section{Limitations}

A retrospective study has its own limitations of generalizability of the study findings to similar high-risk populations and parameters applied in the context of the present analysis. The study was performed at a single tertiary cancer care center; hence, our results might not truly represent other health care models in general public health care settings. Owing to our retrospective design, we were unable to capture other important determinants related to HIV health care parameters including treatment that might impact some of the findings for cervical screening among HIVpositive women. Also, in this retrospective study design, true-positive disease of CIN 2 and above lesions were determined by diagnostic investigation of colposcopy that was offered to all participants and directed biopsies in cases of colposcopic abnormalities. Thus, the possibility of misclassi- fication of disease outcomes due to subjective interpretations in colposcopy cannot be entirely ruled out. However, the retrospective study findings may form the basis for conducting larger longitudinal studies to evaluate low-cost cervical cancer screening technologies and implementation research studies to evaluate the feasibility of implementing cervical cancer screening in STD/HIV/AIDS ICTC in government public health care settings in India.

\section{Conclusions}

Our study's findings strongly highlight the need for cervical cancer screening among the high-risk group of women living with HIV. Adoption and uptake of the cervical cancer screening services by this high-risk group could be improved by integrating the same with STD/HIV testing and counseling clinics established under the National AIDS Control Program. Irrespective of the HIV status of women attending STD/HIV clinics, implementing cervical cancer screening in STD/HIV clinic settings has immense public health implications for increasing the reach for cervical cancer screening. This is due to these clinics providing a single-window opportunity to symptomatic women who are presenting to the clinic, to screen for cervical cancer in the same/single visit since women are unlikely to revisit for preventive screening activities. Also, women receiving ART visit these clinics on a regular basis, which can help the continuity of care needed for cervical cancer screening and referrals for treatment if needed. Our results need replication in STD/HIV/AIDS testing and counseling services settings and if proven generalizable can be included in policy and programmatic implications for integration into these services where they are currently nonexistent.

Acknowledgments

This manuscript has been read and approved by all the authors and represents honest work.

Funding

None.

Conflict of Interest

None declared.

\section{Acknowledgments}

We thank medical social workers, Ms. Parishi Majmudar, Mr. Ashok Patil, and Ms. Bhakti Kabre, for providing preand post-counseling services to HIV-positive women. We are grateful to Mr. Suresh Raibhatanavar and Ms. Nicole Aguirre for helping in data retrieval and analysis. We are thankful to Ms. Rupa Sarwaiya and Ms. Darshana Rane for entering the data.

\section{References}

1 Bray F, Ferlay J, Soerjomataram I, Siegel RL, Torre LA, Jemal A. Global cancer statistics 2018: GLOBOCAN estimates of incidence and mortality worldwide for 36 cancers in 185 countries. CA Cancer J Clin 2018;68(06):394-424 
2 Baseman JG, Koutsky LA. The epidemiology of human papillomavirus infections. J Clin Virol 2005;32(Suppl 1):S16-S24

3 International Agency for Research on Cancer. Globocan India 2020. Accessed December 2, 2021 at: https://gco.iarc.fr/today/ data/factsheets/populations/356-india-fact-sheets.pdf

4 Palefsky J. Human papillomavirus-related disease in people with HIV. Curr Opin HIV AIDS 2009;4(01):52-56

5 Maiman M, Fruchter RG, Clark M, Arrastia CD, Matthews R, Gates EJ. Cervical cancer as an AIDS-defining illness. Obstet Gynecol 1997;89(01):76-80

6 Denny L, Boa R, Williamson AL, et al. Human papillomavirus infection and cervical disease in human immunodeficiency virus-1-infected women. Obstet Gynecol 2008;111(06): 1380-1387

7 Liu G, et al. Prevalent HPV infection increases the risk of HIV acquisition in African women: advancing the argument for HPV immunization. AIDS 2022;36(02):257-265

8 Averbach SH, Gravitt PE, Nowak RG, et al. The association between cervical human papillomavirus infection and HIV acquisition among women in Zimbabwe. AIDS 2010;24(07): 1035-1042

9 Stelzle D, Tanaka LF, Lee KK, et al. Estimates of the global burden of cervical cancer associated with HIV. Lancet Glob Health 2021;9 (02):e161-e169

10 National AIDS Control Organization \& ICMR-National Institute of Medical Statistics. (2020). India HIV Estimates 2019: Report. New Delhi: NACO, Ministry of Health and Family Welfare, Government of India. Accessed September 27, 2021 at: http://naco.gov.in/sites/ default/files/Estimation\%20Report\%202019.pdf

11 Ferreira MP, Coghill AE, Chaves CB, et al. Outcomes of cervical cancer among HIV-infected and HIV-uninfected women treated at the Brazilian National Institute of Cancer. AIDS 2017;31(04): 523-531

12 Chaturvedi AK, Madeleine MM, Biggar RJ, Engels EA. Risk of human papillomavirus-associated cancers among persons with AIDS. J Natl Cancer Inst 2009;101(16):1120-1130

13 Massad LS, Seaberg EC, Watts DH, et al. Long-term incidence of cervical cancer in women with human immunodeficiency virus. Cancer 2009;115(03):524-530

14 Chambuso RS, Shadrack S, Lidenge SJ, Mwakibete N, Medeiros RM. Influence of HIV/AIDS on cervical cancer: a retrospective study from Tanzania. J Glob Oncol 2016;3(01):72-78

15 Shastri SS, Mittra I, Mishra GA, et al. Effect of VIA screening by primary health workers: randomized controlled study in Mumbai, India. J Natl Cancer Inst 2014;106(03):dju009

16 Sankaranarayanan R, Esmy PO, Rajkumar R, et al. Effect of visual screening on cervical cancer incidence and mortality in Tamil Nadu, India: a cluster-randomised trial. Lancet 2007;370 (9585):398-406

17 Sankaranarayanan R, Nene BM, Shastri SS, et al. HPV screening for cervical cancer in rural India. N Engl J Med 2009;360(14): 1385-1394

18 Arbyn M, Sankaranarayanan R, Muwonge R, et al. Pooled analysis of the accuracy of five cervical cancer screening tests assessed in eleven studies in Africa and India. Int J Cancer 2008;123(01): 153-160

19 Sankaranarayanan R, Wesley RS. A practical manual on visual screening for cervical neoplasia. Lyon: International Agency for Research on Cancer, World Health Organization; 2003 IARC Technical Publication; No. 41. Available at A practical manual on visual screening for cervical neoplasia (iarc.fr). Accessed September 27, 2021

20 Nayar R, Wilbur DC. The Pap Test and Bethesda 2014. "The reports of my demise have been greatly exaggerated." (after a quotation from Mark Twain). Acta Cytol 2015;59(02):121-132

21 Darragh TM, Colgan TJ, Thomas Cox J, et al; Members of the LAST Project Work Groups. The lower anogenital squamous terminology standardization project for HPV-associated lesions: back- ground and consensus recommendations from the college of American pathologists and the American society for colposcopy and cervical pathology. Int J Gynecol Pathol 2013;32(01):76-115

22 Dinshaw KA, Tongaonkar HB, Shrivastava SK, Parikh PM, Maheshwari A. Evidence Based Management Guideline. Gynaecological Cancer. Vol III. Mumbai: Tata Memorial Hospital; 2004

23 Bornstein J, Bentley J, Bösze P, et al. 2011 colposcopic terminology of the International Federation for Cervical Pathology and Colposcopy. Obstet Gynecol 2012;120(01):166-172

24 Clifford GM, Gonçalves MA, Franceschi SHPV and HIV Study Group. Human papillomavirus types among women infected with HIV: a meta-analysis. AIDS 2006;20(18):2337-2344

25 De Vuyst H, Lillo F, Broutet N, Smith JS. HIV, human papillomavirus, and cervical neoplasia and cancer in the era of highly active antiretroviral therapy. Eur J Cancer Prev 2008;17(06):545-554

26 Teeraananchai S, Kerr SJ, Amin J, Ruxrungtham K, Law MG. Life expectancy of HIV-positive people after starting combination antiretroviral therapy: a meta-analysis. HIV Med 2017;18(04): 256-266

27 Joshi S, Sankaranarayanan R, Muwonge R, Kulkarni V, Somanathan T, Divate U. Screening of cervical neoplasia in HIV-infected women in India. AIDS 2013;27(04):607-615

28 Chakravarty J, Chourasia A, Thakur M, Singh AK, Sundar S, Agrawal NR. Prevalence of human papillomavirus infection \& cervical abnormalities in HIV-positive women in eastern India. Indian J Med Res 2016;143(01):79-86

29 Gedefaw A, Astatkie A, Tessema GA. The prevalence of precancerous cervical cancer lesion among HIV-infected women in southern Ethiopia: a cross-sectional study. PLoS One 2013;8(12): e84519

30 Hopkins KL, Jaffer M, Hlongwane KE, et al. Assessing national cervical cancer screening guidelines: results from an HIV testing clinic also screening for cervical cancer and HPV in Soweto, South Africa. PLoS One 2021;16(07):e0255124

31 Memiah P, Mbuthia W, Kiiru G, et al. Prevalence and risk factors associated with precancerous cervical cancer lesions among HIVinfected women in resource-limited settings. Aids Res Treat 2012; 2012:953743

32 Teixeira NC, Araújo AC, Correa CM, et al. Prevalence and risk factors for cervical intraepithelial neoplasia among HIV-infected women. Braz J Infect Dis 2012;16(02):164-169

33 Zhang HY, Tiggelaar SM, Sahasrabuddhe VV, et al. HPV prevalence and cervical intraepithelial neoplasia among HIV-infected women in Yunnan Province, China: a pilot study. Asian Pac J Cancer Prev 2012;13(01):91-96

34 Sahasrabuddhe VV, Bhosale RA, Kavatkar AN, et al. Comparison of visual inspection with acetic acid and cervical cytology to detect high-grade cervical neoplasia among HIV-infected women in India. Int J Cancer 2012;130(01):234-240

35 Massad LS, Riester KA, Anastos KM, et al; Women's Interagency HIV Study Group. Prevalence and predictors of squamous cell abnormalities in Papanicolaou smears from women infected with HIV-1. J Acquir Immune Defic Syndr 1999;21(01):33-41

36 Fruchter RG, Maiman M, Sedlis A, Bartley L, Camilien L, Arrastia CD. Multiple recurrences of cervical intraepithelial neoplasia in women with the human immunodeficiency virus. Obstet Gynecol 1996;87(03):338-344

37 Frisch M, Biggar RJ, Goedert JJ. Human papillomavirus-associated cancers in patients with human immunodeficiency virus infection and acquired immunodeficiency syndrome. J Natl Cancer Inst 2000;92(18):1500-1510

38 Joshi SN, Gopalkrishna V, Kumar BK, et al. Cervical squamous intra-epithelial changes and human papillomavirus infection in women infected with human immunodeficiency virus in Pune, India. J Med Virol 2005;76(04):470-475

39 Sankaranarayanan R, Gaffikin L, Jacob M, Sellors J, Robles S. A critical assessment of screening methods for cervical neoplasia. Int J Gynaecol Obstet 2005;89(Suppl 2):S4-S12 
40 Denny L, Anorlu R. Cervical cancer in Africa. Cancer Epidemiol Biomarkers Prev 2012;21(09):1434-1438

41 Akinwuntan AL, Adesina OA, Okolo CA, et al. Correlation of cervical cytology and visual inspection with acetic acid in HIVpositive women. J Obstet Gynaecol 2008;28(06):638-641

42 Palefsky JM. Human papillomavirus-associated anal and cervical cancers in HIV-infected individuals: incidence and prevention in the antiretroviral therapy era. Curr Opin HIV AIDS 2017;12(01):26-30

43 Mwanahamuntu MH, Sahasrabuddhe VV, Blevins M, et al. Utilization of cervical cancer screening services and trends in screen- ing positivity rates in a 'screen-and-treat' program integrated with HIV/AIDS care in Zambia. PLoS One 2013;8(09):e74607

44 Meyerson BE, Sayegh MA, Davis A, et al. Cervical cancer screening in a sexually transmitted disease clinic: screening adoption experiences from a midwestern clinic. Am J Public Health 2015; 105(2, Suppl 2)e8-e14

45 Datta SD, Saraiya M. Cervical cancer screening among women who attend sexually transmitted diseases (STD) clinics: background paper for 2010 STD Treatment Guidelines. Clin Infect Dis 2011;53(Suppl 3):S153-S159 